\title{
A Subject-Independent fMRI-based Neurofeedback System for Depression
}

\author{
Thakkar, Ishani'; Rana, Mohit'; Salinas, Cesar³; Silva, Claudio33; Brett, Claudia4; \\ Pereira, Jaime ${ }^{1}$; Sitaram, Ranganatha ${ }^{5^{*}} ;$ Ruiz, Sergio ${ }^{6^{*}}$
}

${ }^{1}$ Program of Neuroscience, Pontificia Universidad Católica De Chile, Santiago, Chile. ${ }^{2}$ Institute for Medical Psychology and Behavioral Neurobiology, University of

Tübingen, Tübingen, Germany. ${ }^{3}$ Department of Radiology and Imaging, Clínica Alemana de Santiago, Santiago. ${ }^{4}$ Department of Psychiatry, Pontificia Universidad Católica De Chile. ${ }^{5}$ Program of Neuroscience and Department of Psychiatry, Faculties of Biology, Engineering and Medicine, Pontificia Universidad Católica De Chile. ${ }^{6}$ Program of Neuroscience and Department of Psychiatry, Faculty of Medicine, Pontificia Universidad Católica De Chile.

*rasitaram@uc.cl *sruiz@uc.cl

\section{INTRODUCTION AND OBJECTIVES}

Depression, one of the leading causes of disability and death in the world, requires a more robust method of treatment for treatment-resistant patients. Due to imaging techniques like $\mathrm{fMRI}$, real-time neurofeedback (rtNF) has been shown to aid healthy individuals and patients to voluntarily control their hemodynamic activity, leading to observable behavioral changes that can aid rehabilitation in several chronic brain conditions.

Recalling positive emotional experiences acts as endogenous stimulation for positive mood in healthy individuals. In this study, we aim to create a system to train depressive patients to achieve a healthy brain state. We create a subject-independent classifier (SIC) based on healthy brain $\mathrm{fMRI}$ images that were collected during positive autobiographical memory imagery from healthy subjects. We further demonstrate that individuals with depressive symptoms can mimic this healthy brain state using rtNF.

\section{METHODOLOGY}

Whole brain fMRI images from healthy female subjects (1845 years) were collected while performing positive emotional and motor imagery (Fig. 1). The two imageries were used as two classes to provide two distinct classes for differentiation while keeping the classifier robust. Using these images, we created separate age-group-sorted individual-subject SICs using the MANAS4 MATLAB toolbox created by the group that extracts features using a technique called effect mapping that uses mutual information of voxels. One of these SICs (age group 30-35 years) was then used to train a subject with depressive symptoms $(B D I=16)$ using a similar experiment protocol. This subject received real-time neurofeedback calculated from their own functional images while they performed either happy or motor imagery. The classifier differentiated between these two classes to provide positive feedback when their brain state matched the instructed state and negative feedback when it did not. The positive feedback acted as implicit reward to aid the subject in recreating brain states from another individual.
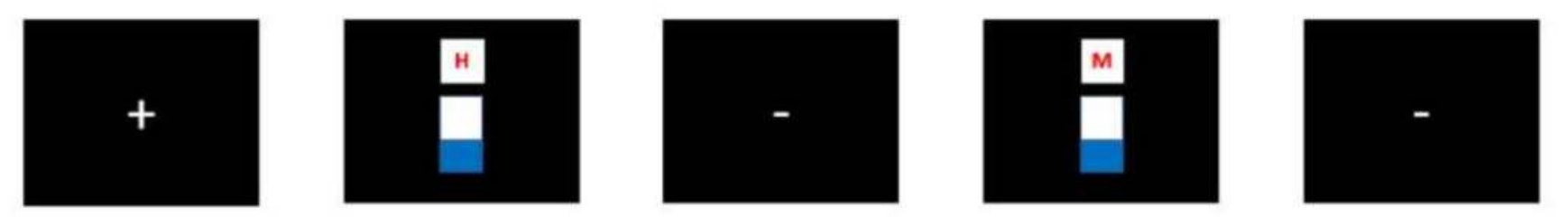

Fig. 1: The figure shows the experiment protocol that appears for both pre-training data collection for building the classifier as well as the training sessions. There is a fixation for the first 10 TRs (each TR is 2 seconds), followed by a 20 TR regulation block and a 5 TR rest. In each run, there are 8 regulation + rest blocks in total, 4 for each condition. Thus, regulation conditions are presented alternatively. The thermometer bar shows the real-time feedback calculated in real time to train subjects with $B D I>10$. In case of healthy subjects, they are informed that the feedback is sham. Each session had 4 such runs.

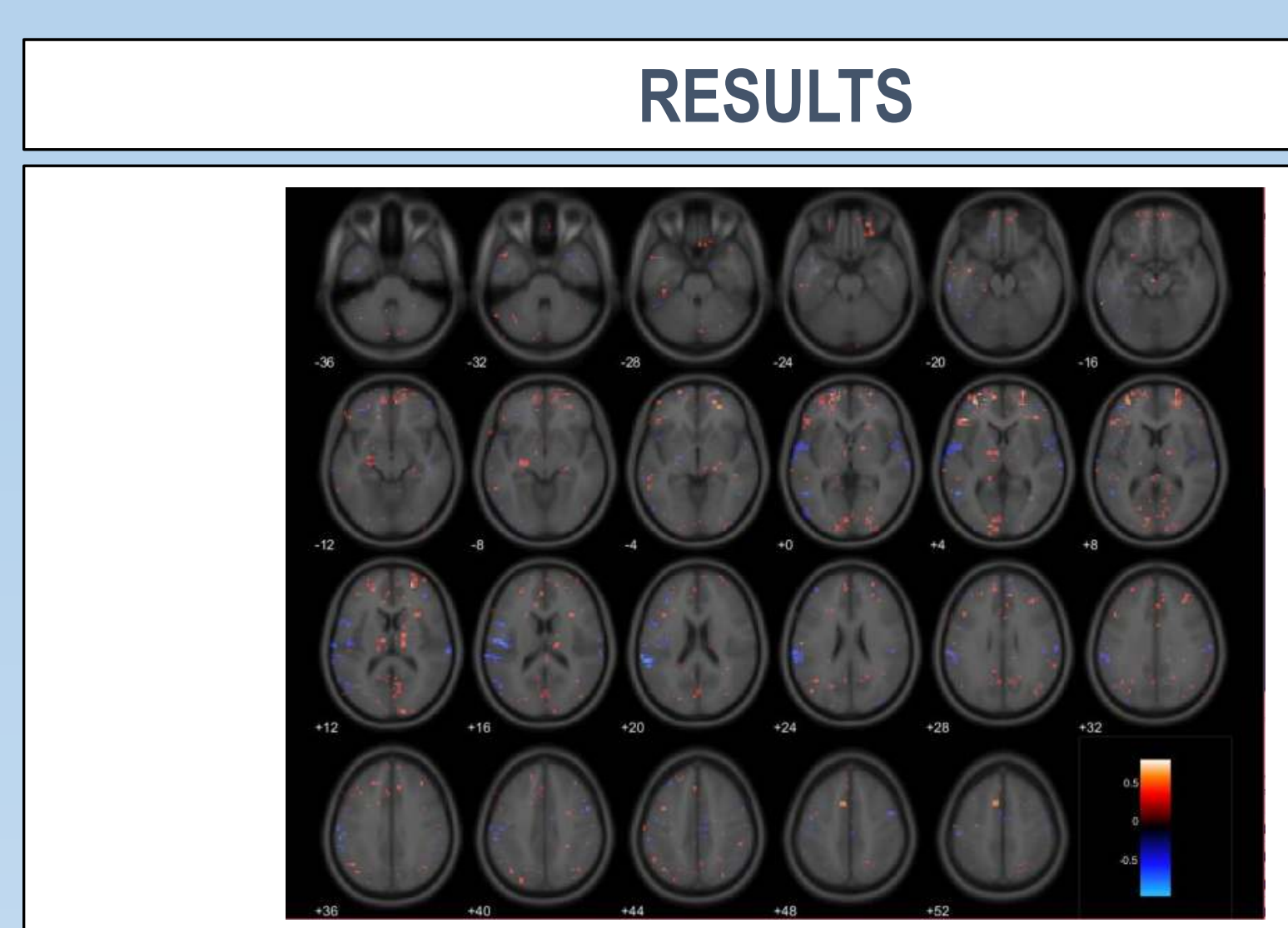

Fig. 2: Most relevant voxels selected as features for classification of brain states for the task extracted from a single healthy subject. The red voxels represent features that were more relevant in happy imagery than in motor imagery, while the blue voxels represent vice versa. Classification accuracy was $90 \%$.

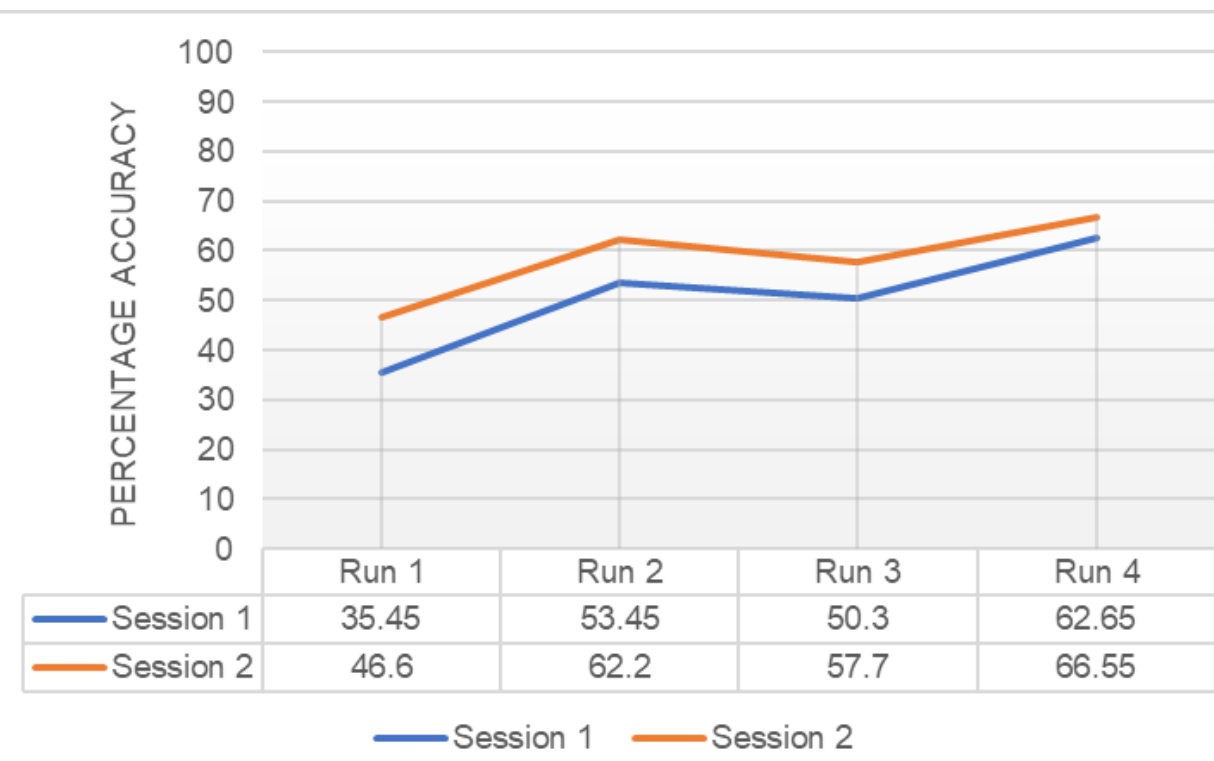

Fig. 3: Performance of a depressive 10 for the SIC training for two sessions across all four runs, as indicated by the classification accuracies of each session.

\section{DISCUSSION}

The classifier created using healthy subject brain images had an accuracy of $90 \%$. The patient with depressive symptoms was able to some extend to "match" the brain patterns of the healthy individual from which the classifier was built.

Learning can be observed with the accuracy increasing within each fMRI run and across both sessions (Fig 3)

Further examinations are being conducted to explore potential clinical benefits of these methodology in depression.

\section{REFERENCES AND ACKNOWLEDGEMENTS}

- Sitaram, Ranganatha, et al. "Real-time support vector classification and feedback of multiple emotional brain states." Neuroimage 56.2 (2011): 753-765.

- Rana Mohit, et al. "A toolbox for real-time subjectindependent and subject-dependent classification of brain states from fMRI signals." Frontiers in Neuroscience 10.3389/fnins.2013.00170

Funded by CONICYT FONDECYT Regular Project $n^{\circ} 1171313, n^{\circ}$ 1171320, CONICYT Anillos Project $n^{\circ}$ ACT172121 